Revue d'histoire de l'Amérique française

REVUE D.HISTOIRE DE L'AMÉRIQUE FRANÇAISE

\title{
Les Gaultier de la Véranderie en France et au Canada et leurs relations par delà l'océan
}

\section{Antonio Champagne}

Volume 12, numéro 2, septembre 1958

URI : https://id.erudit.org/iderudit/301905ar

DOI : https://doi.org/10.7202/301905ar

Aller au sommaire du numéro

Éditeur(s)

Institut d'histoire de l'Amérique française

ISSN

0035-2357 (imprimé)

1492-1383 (numérique)

Découvrir la revue

Citer ce document

Champagne, A. (1958). Les Gaultier de la Véranderie en France et au Canada et leurs relations par delà l'océan. Revue d'histoire de l'Amérique française, 12(2), 262-277. https://doi.org/10.7202/301905ar d'utilisation que vous pouvez consulter en ligne.

https://apropos.erudit.org/fr/usagers/politique-dutilisation/ 


\section{DOCUMENTS INEDITS}

\section{LES GAULTIER DE LA VERANDERIE EN FRANCE ET AU CANADA ET LEURS RELATIONS PAR DELÀ L'OCEAN}

(Documents et commentaires)

\section{EXPLICATIONS PRÉLIMINAIRES}

On peut bien supposer que René Gaultier de Varennes, l'officier de Carignan, lorsqu'il passa les mers en 1665, n'avait pas coupé les liens qui le rattachaient à la mère-patrie.

Le Canada, pendant longtemps encore, restera partie de l'ancienne France, et cela sera vrai, non seulement sur le plan national et officiel, mais autant et plus encore sur le plan familial.

Il se créa des relations de toute sorte entre le nouveau Canadien, qui avait trouvé sur les bords du Saint-Laurent, non pas une patrie nouvelle, mais un prolongement de la mère-patrie, et les personnes et le pays qu'il avait quittés mais qui restaient toujours les siens. Ses enfants et souvent même ses petits-enfants n'eurent pas d'autres sentiments, et les relations continuèrent pendant deux générations au moins, quoique à un rythme qui se ralentissait graduellement.

Combien l'histoire de nos familles serait différente, surtout celle de nos grands hommes, combien elle serait plus complète et plus vraie, si l'on pouvait suivre les rapports qui s'établirent ainsi par delà l'océan!

Par bonheur, nous avons pu, après de longues et patientes recherches sur les Gaultier de Varennes et de La Vérendrye, mettre la main sur plusieurs documents qui nous font revivre partiellement l'histoire de cette intéressante famille, et ce sont ces documents que nous allons livrer au lecteur, en les accompagnant des commentaires jugés nécessaires.

Les détails généalogiques qui suivent nous permettront à la fois de rattacher les différentes branches de la famille à un 
même tronc et d'identifier les personnages nommés dans les pièces dont nous donnerons le texte. ${ }^{1}$

Un René Gaultier, qui ne porte aucun titre dans les documents connus et qui semble n'en avoir jamais eu, épouse en premières noces, vers 1550, Jehanne Guespin, et donne naissance, par ses fils Jacques et René, aux Gaultier de La Blanchardière et aux Gaultier de Boumois.

Ces deux branches s'éteignirent au $17^{\top}$ siècle.

Marié en secondes noces, vers 1569, à Perrine Froger, il est l'auteur, par son fils François, de la branche de Longlée, qui disparaît comme les deux premières, puis, par son fils Pierre, de celle des La Veranderie, qui se perpétuera au Canada, après s'être éteinte à son tour dans l'ancienne France.

Né à Angers, dans la paroisse de Saint-Michel-du-Tertre, Pierre y est baptisé le 27 février 1576, et il décède entre le 4 février et le 30 novembre 1619 . On le trouve avec le titre de «La Veranderie » le 27 août 1597. C'est la première mention de ce nom illustre dans les documents connus.

Marié vers 1600 à Renée Jarry, Pierre Gaultier eut dix enfants au moins, dont un seul, Adam-Pierre, laissa une descendance masculine.

Parmi les autres, voici ceux qui nous intéressent surtout et dont les noms reviennent dans les documents, particulièrement ceux dont nous donnons les textes ci-après:

- Catherine, née entre 1600 et 1603 , mariée en premières noces, en 1619, à Pierre Lecomte et en deuxièmes noces à Anastase Lemantel. Elle eut, de ce dernier; Charles, - Anne, mariée à César-Charles Boétey, - et Marie.

- Jacques, 1606-1671, abbé de la puissante abbaye de Pontron, en Anjou.

- Hélène, 1612-1694, célibataire.

- Claude (fille), 1614-1687, célibataire.

\footnotetext{
1 On trouvera des détails supplémentaires sur la famille Gaultier: généalogie, terres et titres, état social, armoiries, dans les articles que nous avons publiés dans le Bulletin des Recherches Historiques d'avril-mai-juin et d'octobre-novembre-décembre 1956.
} 
Adam-Pierre Gaultier de La Veranderie, deuxième du nom, baptisé à Saint-Michel-du-Tertre, Angers, le 19 novembre 1604, décédé à La-Cour-du-Tremblay, près de Bécon, et enseveli dans cette ville le 10 octobre 1670, épousa entre 1624 et 1632 Bertrande Gourdault et eut d'elle au moins les cinq enfants suivants, dont nous n'avons pu retracer les actes de baptême:

- Charles, aîné, sieur de La Veranderie, troisième du nom, devenu gouverneur d'Arlon, dans les Flandres, et décédé en cette ville le 26 février 1697.

- René, sieur de Varennes, l'officier de Carignan, qui épousa en 1667 Marie Boucher et devint l'auteur de la branche canadienne.

- Perrine, qui épousa en premières noces, en 1663, PierrePaul Philibert de l'Ingranderie. Elle en eut plusieurs enfants, dont Charles-Gabriel, né vers 1674, qui fit du service au Canada depuis 1706 au moins, en qualité d'officier, décéda à Québec le 4 septembre 1734, au retour d'un voyage en France, et y fut enseveli le même jour; et sa sœur, nommée Jacquette, née vers 1665, décédée en 1707.

Perrine épousa en secondes noces, vers 1673, Pierre Lebœuf de Beauregard, et en eut un fils, Pierre, né en 1674.

- Renée, mariée à André Ramauger, sieur de La Sablonnière, dont elle eut un fils Bernard.

- Marie, qui épousa à Cheillé, (Indre-et-Loire), ancienne Touraine, le 12 juillet 1701, Pierre Carcat, sieur Dupré et de La Roche.

Charles Gaultier de La Veranderie, l'aîné d'Adam-Pierre et de Bertrande Gourdault, étant mort sans enfants, la famille disparaît en France, et c'est le cadet, René Gaultier de Varennes, qui continue la lignée au Canada.

Ayant marié aux Trois-Rivières, le 26 septembre 1667, Marie Boucher, fille du célèbre Pierre Boucher, il en eut dix enfants, dont l'aîné, Louis, sieur de La Veranderie, quatrième du nom, et Pierre, le quatrième des fils, sieur de Boumois, puis de La Veranderie ou La Vérendrye, "Découvreur » de l'Ouest, nous intéressent ici d'une façon particulière. 
René Gaultier de Boumois, premier du nom, frère de Pierre Gaultier de La Veranderie, mentionné plus haut, eut un fils, Louis Gaultier de Boumois puis de Varennes, que nous retrouverons également.

Tels sont les noms qui reparaîtront dans les documents et les explications que nous donnerons ci-après.

Tous ceux qui s'intéressent à l'histoire des Gaultier de Varennes et de La Vérendrye ont une vague connaissance de certains faits qui ont été signalés dans les livres et les revues et dont l'exposé peut se résumer ainsi :

1. René Gaultier de Varennes se rend en France en 1671 et en revient en 1672. Il veut y retourner en 1688-1689, mais la mort l'empêche de réaliser son désir. - Quelles ont pu être les raisons de tels voyages?

2. Louis Gaultier de La Veranderie, son fils aîné, passe en Europe comme soldat entre 1693 et 1703 , et y meurt entre 1706 et 1711, alors qu'il était capitaine dans le régiment de Bretagne.

3. Pierre, son frère, le futur découvreur de l'Ouest, part à son tour en 1707, avant ou après le décès de Louis, se bat dans les Flandres, est blessé grièvement à Malplaquet en 1709, est fait lieutenant sur le champ de bataille, revient au Canada en 1711 ou 1712, simple enseigne selon les uns, sans aucun grade militaire selon les autres.

4. Pour des raisons que nous ignorons, Pierre cherche à repasser en France en 1724-1727. - A-t-il fait ce voyage ?

5. Un oncle ou grand-oncle de La Vérendrye, nommé Charles, fut gouverneur d'Arlon, dans les Flandres, et on le trouve mentionné dans des documents canadiens par rapport à certaines sommes d'argent.

6. Un cousin de La Vérendrye, Charles-Gabriel Philibert, mourut à Québec en 1734, au retour d'un voyage en France.

Là s'arrêtaient jusqu'à présent nos connaissances sur les rapports qui ont pu exister entre les Gaultier du Canada et la mère-patrie. 
La présente étude, en plus de rectifier certaines inexactitudes contenues dans le précédent exposé, le précise et le complète; mais elle ne se contente pas de cela, car elle apporte aux chercheurs et aux historiens une importante série de textes originaux qui jetteront un nouveau jour sur cette intéressante famille et en particulier sur le futur découvreur de l'Ouest.

Parmi les renseignements que nous avons à présenter, les uns se rapportent surtout, mais non uniquement, à des affaires matérielles et à des questions de succession; les autres concernent plus particulièrement la carrière militaire de certains membres de la famille.

I. René Gaultier de Varenines Passe en France en 1671 et VEUT Y RETOURNER EN 1688-1689.

René Gaultier de Varennes était venu au Canada en 1665, avec le régiment de Carignan et en qualité de lieutenant dans la compagnie d'Arnoult de Broisle, sieur de Loubias.

Le régiment ayant été congédié en 1667 , René décida de rester au Canada, conformément aux désirs manifestés par le roi.

Dès le 5 avril 1666, en effet, Colbert écrivait à Talon que le roi avait été bien aise d'apprendre qu'un grand nombre d'officiers et de soldats de Carignan avaient manifesté le désir de s'établir au pays, pour peu qu'on les aidât financièrement. Il ajoutait que le roi serait heureux que tous les autres fissent la même chose. ${ }^{2}$

Le 22 septembre 1667, c'était chose faite pour ce qui concerne René Gaultier de Varennes, puisqu'il passait son contrat de mariage avec la fille du gouverneur des Trois-Rivières, Marie Boucher. $^{3}$ Le mariage eut lieu quatre jours plus tard, dans cette localité. $^{4}$

\footnotetext{
Abréviations:

AN - Archives Nationales, Paris.

BN - Bibliothèque Nationale, Paris.

BRH - Bulletin des Recherches Historiques, Québec.

RAPQ - Rapport de l'Archiviste de la province de Québec, Québec.

2 BN. Manuscrits français. Nouvelles acquisitions, $\mathrm{N}^{\circ} 9269$, folio 424 . - Publiée dans le Rapport de l'Archiviste de la province de Québec, 19301931, p. 45.

3 Greffe de Sévérin Ameau, Cour Supérieure des Trois-Rivières.

4 Registre des baptêmes, mariages et sépultures de Notre-Dame des Trois-Rivières.
} 
René Gaultier fut bientôt nommé gouverneur de la place, en remplacement de son beau-père, qui désirait se retirer dans sa seigneurerie des Iles-Percées ou Boucherville, afin d'y installer une colonie modèle.

Le changement eut lieu entre la mi-avril et le 20 mai $1668 .^{5}$

Mais voici que, à l'automne de 1671, l'idée vient à René Gaultier de faire un voyage en France.

Il nous est permis de nous demander quelle raison il pouvait avoir. Le roi, en effet, regardait d'un mauvais œil ces longues absences qui pouvaient nuire à la Colonie de plusieurs façons, tant que celle-ci n'était pas établie plus solidement.

Aussi l'intendant Talon s'explique-t-il dans un document bien connu, daté du 2 novembre 1671, dont nous donnons ici l'essentiel: «Plusieurs officiers se déterminaient, dit-il, à repasser en France pour y traiter leurs affaires et retourner ensuite. J'ai fait ce que j'ai pu pour les en divertir... Tous ont déféré à ce sentiment, un excepté, qui s'est vu indispensablement engagé à faire le voyage. Comme il est marié... ayant le gouvernement des Trois-Rivières, le retour en est sûr ... ${ }^{6}{ }^{6}$

René était encore au Canada le 23 octobre 1671, puisque, ce jour-là, il reçoit de $M$. de Courcelle, gouverneur général, un certificat de ses services qui pouvait lui être utile en cours de route. ${ }^{7}$ Il a pu partir le 2 novembre, date de la lettre de l'intendant, ou un des jours suivants.

${ }^{5} \mathrm{Au}$ contrat de mariage du 22 septembre 1667, Pierre Boucher s'engage à nourrir sa fille et son gendre pendant six mois, ce qui mène au 22 mars 1668. Ensuite, il fallut sans doute attendre que le Saint-Laurent, la seule route praticable d'alors, fût libre de glaces, ce qui ne pouvait guère se produire avant la fin d'avril.

Le 20 mai suivant, la famille devait être rendue à Boucherville, puisque, au baptême d'une petite sauvagesse ce jour-là, on a pour parrain et marraine Ignace et Marie Boucher. Ignace est évidemment le fils de Pierre Boucher, alors âgé de 9 ans. Quant à Marie, elle pouvait être, ou bien la sour de Pierre Boucher, veuve d'Etienne Pépin dit Lafont, ou bien sa fille, épouse de René Gaultier.

6 Talon à Colbert. AN. Colonies, C $11 \mathrm{~A}$, Correspondance générale, vol. 3: 1668-1672, fol. 202; - et Archives du Ministère des Affaires Etrangères, Paris. Amérique. Vol. 2: 1661-1668, fol. 279.

7 Mentionné dans l'Inventaire de René Gaultier de Varennes par Antoine Adhémar, 1er juillet 1693. - Voir: RAPQ (1949-1950 et 1950-1951), 44. 
Nous n'en savons guère plus long sur ce voyage, sauf que, le 6 juin 1672, René Gaultier était nommé gouverneur des TroisRivières pour un second terme, ${ }^{8}$ non plus cette fois par le gouverneur de Québec, mais par le roi lui-même, sur la recommandation de Talon. De plus, la lettre de nomination renfermait des formules élogieuses propres à rehausser son prestige. René revint au Canada avec cette nouvelle auréole. Cette nomination sera renouvelée le 8 mars $1675,{ }^{9}$ et de trois en trois ans jusqu'à sa mort en 1689.

Mais ce n'était pas là le but du voyage. René avait déjà toutes les sympathies, et sa nomination par le roi n'était qu'une affaire de routine. On s'est demandé et avec raison quels pouvaient être les motifs qui l'animaient.

Or, nous croyons pouvoir donner une explication plus que vraisemblable.

Adam-Pierre Gaultier de La Veranderie, père de René, était mort à La-Cour-du-Tremblay et avait été enseveli à Bécon le 10 octobre 1670. ${ }^{10}$ La nouvelle du décès dut parvenir au Canada par les bateaux de 1671, qui, selon l'habitude, avaient dû partir en avril et arriver en juin.

Il n'est pas difficile de comprendre pourquoi René Gaultier s'était « vu indispensablement engagé à faire le voyage », selon l'expression de Talon.

René Gaultier était pauvre. Ses émoluments de 1200 livres par année comme gouverneur des Trois-Rivières ne lui suffisaient pas à élever sa famille, à tenir son rang de gouverneur, et à recevoir tous les personnages qui évoluaient entre Québec et les TroisRivières. ${ }^{11}$ Il fallait d'autres sources de revenus.

8 Provisions de gouverneur des Trois-Rivières pour le sieur de Varennes. - Saint-Germain-en-Laye. AN. Colonies, B 4, fol. 71 et 72.

9 Copie de cette nomination au greffe d'Ameau. - Entrée par erreur au 31 octobre 1672. - Cour Supérieure des Trois-Rivières.

10 On trouvera son acte de décès dans l'article que nous avons publié dans le BRH d'octobre-novembre-décembre 1956.

11 Dans les toutes dernières années, c'est-à-dire après 1685 , il aura 3000 livres, mais il devra maintenir à ses frais une garnison de huit hommes, comprenant un officier et sept soldats.

Voir: a) Lettre de l'intendant De Meulles, du 28 septembre 1685. AN. Colonies, C 11 A, Corr. gén., vol. 7, fol. 143;

b) Requête de Marie Boucher, veuve de Varennes, au Ministre, (16901691), BN. Mss. français. Nouv. acquis. Margry, No 9386, p. 14. 
Un peu plus tard, il fera la traite des fourrures dans sa concession de La Gabelle, sur la rivière des Trois-Rivières, devenue le Saint-Maurice, mais cette concession ne lui fut octroyée qu'en 1673, et il ne semble pas que le gouverneur des TroisRivières y ait fait fortune.

Car on a la preuve que René s'endettait.

Pour le moment, il allait recueillir la part qui lui revenait de la succession paternelle, qui lui était si nécessaire. Il en profitait pour revoir sa famille et son pays d'Anjou, en même temps que pour travailler à ses intérêts à la Cour, avec la recommandation de Talon et celle de M. de Courcelle: * Cet officier, nommé Varennes, a du mérite et ne manque pas de zèle ». En revint-il plus riche? Il y a lieu d'en douter. Nous ne trouvons rien dans les documents.

Cette explication des motifs du voyage que fit René Gaultier en 1671 est d'autant plus plausible qu'en 1688-1689, le gouverneur des Trois-Rivières renouvelle sa demande dans une circonstance analogue. Il s'agit de la mort de sa tante Claude Gaultier, décédée à Paris le 20 juillet 1687, et d'une nouvelle affaire de succession. La nouvelle de la mort dut arriver au Canada en juin 1688. René fit sa demande. Le roi refusa d'abord: on sait que le temps n'était pas propice. Les Iroquois avaient repris leurs incursions: - on allait déplorer, quelques mois plus tard, le massacre de Lachine et bien d'autres faits tragiques. - Le $1^{\text {er }}$ mai 1689 , le Ministre écrivait donc à M. de Denonville qu'il « ne croit pas devoir accorder, dans les conjonctures actuelles, au sieur de Varennes, gouverneur des Trois-Rivières, le congé qu'il demande. ${ }^{12}$ Mais il se ravise bientôt et, le 24 mai, la «permission » est accordée \& à M. de Varennes, gouverneur des Trois-Rivières, de passer en France pour y vaquer à ses affaires, avec ordre de repasser à son gouvernement par les premiers vaisseaux qui repartiront pour ce pays en $1690 \gg^{13}$

Le voyage se fit dans une direction imprévue: quand la permission arriva au Canada, René était mort depuis le 4 juin. 
Le document suivant va nous renseigner sur les circonstances de la nouvelle demande de René Gaultier et nous donner d'intéressantes informations sur la famille en France.

\section{Partage De Demoiselle Claude Gaultier: 7 Janvier 1688.}

Pièce $\mathrm{N}^{\circ} 1$.

(Partage de demoiselle Claude Gaultier. - Despriez et Thibert, notaires, - Paris, 7 janvier 1688. - Archives Nationales, Paris. Minutier central. Etude 51, liasse 634).

«Pardevant les notaires garde-notes du Roy au Châtelet de Paris, soussignés, furent présents:

« Charles Gaultier, écuyer, seigneur de La Veranderie, gouverneur de Marche, y demeurant ordinairement,... au nom et comme procureur de damoiselle Hélène Gaultier, fille majeure, sa tante, héritière pour un tiers de défunte damoiselle Claude Gaultier, sa sœur, aussi fille majeure, fondé de sa procuration (d'Hélène), passée pardevant Huault, notaire royal en Touraine, résidant en la ville de Fay, ressort de Chinon, le 8 août dernier (1687) ...;

«Damoiselle Renée Gaultier, femme du sieur André Ramauger, bourgeois de Rouen, en son nom et comme procuratrice dudit sieur son mari..., autorisée par procuration passée pardevant Pichon et Sanadon, notaires de Rouen, le 10 décembre dernier..., la dite damoiselle demeurant ordinairement au dit Rouen...;

«Philippe Desnoyers, bourgeois de Paris, comme procureur de Pierre Lebœuf, sieur de Beauregard, et damoiselle Perrine Gaultier, sa femme, et de damoiselle Marie-Renée, fille majeure, fondé de leurs procurations: celle des dits sieur et damoiselle de Beauregard pardevant Dovalle et Amat, notaires royaux à Saumur, le 27 décembre dernier, - et celle de damoiselle Marie Gaultier pardevant Huault, notaire en Touraine, le 29 du dit mois,... et encore icelui Desnoyers se faisant fort de René Gaultier, écuyer, sieur de Varenne, gouverneur des Trois-Rivières en Canada, auquel il promet faire ratifier les présentes ... dans un an;... ladite damoiselle Ramauger et (damoiselle) de Beauregard, sieur de Varenne, et damoiselle Marie-Renée Gaultier héritiers de défunt Pierre Gaultier, écuyer, sieur de la Veranderie, leur père, pour un tiers de ladite défunte damoiselle Claude Gaultier, leur tante, d'autre part;

* Et Charles Lemantel, écuyer, seigneur et patron de Moyaux, Chipin et Les Cheminées, demeurant en sa terre dudit Moyaux, proche Lizieux, tant en son nom que comme procureur de César-Charles Boétey, seigneur du Buisson et de Grand Vau et de dame Anne Lemantel, son épouse, et de damoiselle Lemantel, fille majeure,... fondé de deux procurations passées, celle desdits sieur et dame du Grand Vau pardevant Robert du Val, notaire 
royal à Orbec, et Laurent Heuze, son adjoint, le 17 août dernier, et celle de ladite damoiselle Lemantel pardevant Jouan, notaire en la vicomté d'Orbec pour le siège de Blangy, sans date,... lesdits sieur de Moyaux, dame du Grand Vau et damoiselle Marie Lemantel, héritiers pour l'autre tiers de défunte damoiselle Catherine Gaultier, leur mère, au jour de son décès veuve d'Anastase Lemantel, vivant seigneur et patron dudit Moyaux, de ladite défunte et damoiselle Claude Gaultier leur tante encore d'autre part, lesquels ont dit que le décès de ladite damoiselle Claude Gaultier étant arrivé, scellé aurait été à l'instant apposé sur les biens par Me Nicolas Labbé, commissaire examinateur au Châtelet de Paris, et ensuite fait l'inventaire par Me Benoist et son collègue, notaires à Paris, les 29 juillet et jours suivants de l'année dernière 1687, à la requête de messire Jean Angot, seigneur de la Motte, Conseiller du Roy en sa Cour de Parlement en Normandie, et de messire Antoine-François Lefebvre d'Ormesson, chevalier, Conseiller du Roy en ses Conseils,... curateur du dernier des testaments et codicilles à l'olographe de ladite damoiselle Gaultier, en date du 2 août 1686 (date du testament), (inventaire fait) en présence de Me Antoine Dupeyron, Conseiller du Roy, substitut de M. le Procureur de Sa Majesté au Châtelet, pour l'absence desdits sieurs, dames et damoiselles héritiers; par lesquels testaments et codicilles ladite damoiselle Gaultier aurait fait plusieurs dispositions, lequel dernier testament a été seul exécuté, suivant la transaction faite entre lesdits sieur de la Veranderie, procureur tant de ladite damoiselle Hélène Gaultier que desdits sieurs et damoiselle Ramauger et de Beauregard et damoiselle Marie-Renée Gaultier et s'étant fait fort dudit sieur de Varenne et sieur de Moyaux, - et la plus grande partie des héritiers de ladite défunte damoiselle Gaultier, (transaction passée) pardevant Despriez et Thibert le 15 décembre dernier, conformément à laquelle transaction délivrance a été faite, tant par ledit sieur de la Veranderie et (sieur) de Moyaux que par ledit seigneur d'Ormesson des legs portés audit testament, - deniers comptants, meubles, vaisselle d'argent, fonds de rente et pensions viagères de 250 livres par an qui étaient payables aux Pères Forget et Langeron, religieux de la maison Saint-Antoine de cette ville, lesquels sont exécutés au moyen de la compensation que lesdits sieurs de la Veranderie et de Moyaux ont faite jusque concurrence de 5000 livres en déduction de 8000 faisant le principal de 333 livres, 6 sols, 8 deniers de rente au denier vingt-deux ${ }^{14}$ qui étaient dus à la succession de ladite damoiselle Gaultier par ladite maison Saint-Antoine, ayant lesdits sieurs de la Veranderie et de Moyaux reçu les 3000 livres de surplus...; après la délivrance de tous lesquels legs et le paiement fait par ledit seigneur

14 L'intérêt de l'argent, au $17^{\mathrm{e}}$ et $18^{\mathrm{e}}$ siècles, était presque invariablement d'un sou par livre, ou 1/20, ou 5\%. On disait: \& Au denier vingt $\gg$.

Comme on se servait aussi parfois de livres de 22 sous, et que l'intérêt était encore d'un sou par livre, il se trouvait alors de 1/22 ou un peu plus de 41/2. On disait dans ce cas: «Au denier vingt-deux ». 
d'Ormesson des frais funéraires, messes et prières ordonnés par ladite défunte, ensemble les frais de scellé et inventaire, icelui seigneur d'Ormesson a rendu compte de l'exécution dudit testament, et auxdits sieurs, dames et damoiselles héritiers, a été entre lui et lesdits sieurs de la Veranderie et de Moyaux sous leurs seings le 5e du présent mois, reconnu le même jour pardevant Thibert et son collègue, par la fin duquel compte s'étant trouvé rester entre les mains dudit seigneur d'Ormesson la somme de 15742 livres,... sols 15 , il aurait remis ladite somme aux sieurs de la Veranderie et de Moyaux, conjointement avec quelques bourses de jetons restées de celles contenues audit inventaire, ensemble tous les titres et papiers qui restaient de ceux inventoriés,... lesquelles bourses et jetons d'argent iceux sieurs de la Veranderie et de Moyaux ont vendu la somme de 519 livres, 15 sols, qu'ils ont reçus.

* Et désirant lesdits sieurs, dames et damoiselles héritiers jouir divisement des biens qui restent de la succession de ladite damoiselle Gaultier, ils sont convenus qu'ils consistent:

* Premièrement en la somme de 15742 livres, 5 sols, reliquat du compte d'exécution testamentaire;

* plus 519 livres, 15 sols, vente des bourses de jetons d'argent;

* plus 150 livres reçues par les sieurs de la Veranderie et de Moyaux du sieur de la Motte, legs particulier de la damoiselle Gaultier, pour les causes portées dans la transaction du 15 décembre dernier;

* plus 179 livres, 10 sols touchés par les sieurs de la Veranderie et de Moyaux, du nommé Payen, maître tonnelier, pour loyers échus jusqu'au 20 de juillet dernier (1687), jour du décès de ladite damoiselle Gaultier, de la petite maison qu'il occupait rue des Juifs, joignant rue Grande, qui a appartenu à icelle damoiselle et par elle donnée à mademoiselle de la Motte, par donation entre vifs, avec réserve d'usufruit au profit de ladite damoiselle Gaultier;

«plus la somme de 244 livres, faisant partie de celle de 250 livres, reçue par les sieurs de la Veranderie et de Moyaux pour les derniers six mois de l'année dernière 1687, de 500 livres de rente sur les Aydes et Gabelles qui étaient de la succession et qui ont été délaissées audit sieur de la Veranderie en son nom pour le legs de famille, somme de 500 livres de rente à lui faite par ladite damoiselle Gaultier, suivant l'acte passé pardevant Thibert et son collègue le 5 du présent mois, les six livres de surplus ayant été retenues par le sieur de Jumentier pour son droit de recette;

* plus 3000 livres reçues desdits religieux de Saint-Antoine pour le reste du principal de 333 livres, 6 sols, 8 deniers de rente qu'ils doivent à la succession.

*plus 150 livres de rente rachetable de la somme de 3000 livres constituée par défunt Me Pierre Lefebvre Delezeau, Conseiller du Roy en

15 Lire: « 5 sols $\gg$. 
sa Cour des Aydes au profit de la damoiselle Claude Gaultier, par contrat passé pardevant Dionis et Benoist le 6 novembre 1679;

«plus 300 livres de rente au principal de 6000 livres constituée à la damoiselle Claude Gaultier pour les sieurs et dame de la Motte sous signature privée du $1^{\text {er }}$ juillet 1686 ;

\&plus 601 livres, 7 sols restant dus des arrérages desdites deux parties de rente et de plusieurs viagères payables durant la vie de ladite damoiselle Gaultier jusqu'à son décès et desdites deux rentes jusqu'au dernier de décembre 1687;

« Somme totale de tous lesdits biens, 29436 livres, 17 sols, qui est, pour chacun tiers desdits sieurs, dames et damoiselles et participants: 9812 livres, 5 sols, 8 deniers;

(On donne ici la façon de partager dans le détail les sommes susdites, particulièrement:)

$\ll \grave{A}$ chacun desdits damoiselles Ramauger et de Beauregard, sieur de Varenne et damoiselle Marie-Renée Gaultier, 2453 livres, 1 sol, 5 deniers... (formant en tout un tiers de la succession de 9812 livres, 5 sols, 8 deniers) ...

\& À la charge de contribuer chacun aux frais...

* Car ainsi a été accordé et convenu entre lesdites parties...

《 Fait et passé à Paris en la maison dudit Thibert, notaire, le 7e jour de janvier 1688. Ladite damoiselle Ramauger a déclaré ne savoir écrire ni signer et les autres parties ont signé:

« Gaultier de La Veranderye - Charles Lemantel - Desnoyers Despriez - Thibert ».

Ce long document ne demande pas d'explications compliquées. L'essentiel pour nous, à savoir la partie concernant les personnes, le montant total de la succession et la part revenant à chacun, est parfaitement clair.

Nous ferons seulement deux remarques, qui résumeront tout ce qu'il importe vraiment de savoir:

$1^{\circ}$ Les personnes nommées dans l'acte constituent la famille entière des Gaultier de la Veranderie, tant en France qu'au Canada, c'est-à-dire les enfants et petits-enfants de Pierre Gaultier et de Renée Jarry, l'auteur de la lignée, au 7 janvier 1688.

Ce sont, après la mort de Claude: a) Hélène, fille majeure ; - b) les cinq enfants vivants d'Adam-Pierre: Charles Gaultier de La Veranderie, gouverneur de Marche-en-Famenne et plus tard d'Arlon; - René Gaultier de Varennes, époux de Marie Boucher et gouverneur des Trois-Rivières; - Perrine, épouse de Pierre Lebœuf de Beauregard après l'avoir été de Pierre-Paul Philibert; - Renée, épouse d'André Ramauger; - Marie, fille 
majeure, qui épousera Pierre Carcat en 1701 ; - c) les enfants vivants de Catherine, mariée à Anastase Lemantel, lesquels sont: Charles, - Anne, mariée à César-Charles Boétey, - Marie. De son premier mari, Pierre Lecomte, Catherine n'a pas d'enfants en 1688.

Nous n'avons pas à nous occuper ici de la génération la plus jeune, comprenant les enfants de René Gaultier de Varennes, ceux de Pierre-Paul Philibert, ceux de Pierre Lebœuf et ceux de Ramauger, dont Pierre Lebœuf, fils, et Bernard Ramauger, seront mentionnés à Arlon et 1697 , - et peut-être d'autres descendants de Catherine par son époux Lemantel.

$2^{\circ}$ Pour ce qui concerne la succession de damoiselle Claude, nous voyons qu'Hélène, seule survivante des enfants de Pierre Gaultier de La Veranderie et de Renée Jarry, recevra un tiers, - les enfants d'Adam-Pierre, pris dans leur ensemble, en auront un autre, et ceux de Catherine se partageront le troisième.

La succession totale consiste en une somme de 29436 livres et 17 sols, qui sera divisée en trois parts de 9812 livres, 5 sols et 8 deniers chacune.

Des cinq enfants d'Adam-Pierre, qui nous intéressent surtout, Charles est hors de cause, ayant déjà reçu avant la mort de sa tante et pour des raisons spéciales que nous ignorons, une rente annuelle de 500 livres, représentant un capital de 10000 livres.

Les quatre autres recevront chacun un quart du tiers, ou un douzième, de 29436 livres et 17 sols, soit 2453 livres, 1 sol et cinq deniers. Telle est la part de René Gaultier de Varennes, ou plutôt de ses enfants, pris dans leur ensemble.

Pour éviter les contestations et difficultés de toute sorte, un arrangement est fait, selon lequel telle ou telle des sommes constituant la succession ira à tel héritier plutôt qu'à tel autre. Nous n'avons pas donné le texte, qui n'a pas d'importance pour nous.

Enfin, les quelques frais qu'entraîne l'exécution du testament et autres mesures légales seront partagés entre tous, au prorata de la part reçue.

Tel est le contenu de cet important document du partage de damoiselle Claude Gaultier. 
2400 livres à partager entre René, sa femme et les neuf enfants qu'ils avaient à la date du 7 janvier 1688, cela ne faisait certes pas une fortune pour chacun ! Encore verrons-nous qu'ils n'avaient rien touché de cette somme en avril 1697.

Le document qui suit règle la question:

\section{LOUIS GAULTIER DE LA VERANDERIE PASSE EN FRANCE EN 1693 ET MEURT SUR LE CHAMP DE BATAILLE EN 1706-1707}

On a été très embarrassé jusqu'à présent pour fixer la date du départ de Louis Gaultier de La Veranderie pour la France. Les chiffres donnés ont évolué entre 1693 et 1703 , ou même après.

Pièce No 2.

(Archives Nationales, Paris. - Colonies. - D2C, 47, fol. 73).

« Rôle des officiers qui servent en Canada ... (Par Champigny, Québec, 6 novembre 1693).

« Places vacantes par l'absence des nommés ci-après, passés en France et avancés ...

«... La Verandrye. Passé en France, lieutenant réformé...».

Nous voilà donc fixés d'une façon passablement précise sur la date de ce départ: automne de 1693.

Comme les bateaux réguliers quittaient généralement Québec pour la France vers la fin d'octobre ou la première quinzaine de novembre, on peut dire que Louis La Veranderie quitta le Canada à cette époque de l'année, mais avant le 6 novembre de l'année 1693.

Louis dut se rendre tout d'abord visiter sa famille de France, qu'il ne connaissait pas, et faire connaissance avec les lieux d'Anjou qu'avait fréquentés son père.

Puis il alla rejoindre son régiment.

Dès qu'il le put, il donna des nouvelles à sa famille. Nous en trouvons des échos dans un mémoire de Pierre Boucher, son grand-père, dans lequel le fondateur de Boucherville donne des 
détails précis sur lui-même et sa famille. ${ }^{16}$ Ce mémoire n'est pas daté, mais il est facile, d'après son contenu, de l'attribuer à l'année 1695. En voici quelques extraits:

« Je suis un des plus anciens du pays, y ayant été amené par feu mon père en 1635. J'étais pour lors âgé de 13 ans... J'ai eu neuf garçons, six filles ... L'aînée a été mariée à M. de Varennes, mort gouverneur des Trois-Rivières. Son frère, nommé Monsieur de Veranderie, est gouverneur d'Arlon, et son fils, qui s'appelle de Varennes, est en France, cornette (enseigne) dans les Dragons... Sa fille est mariée à M. de Levilliers, capitaine... Voilà la $60 \mathrm{e}$ année que je suis dans le pays et j'en ai $73 \ldots$

Ces renseignements sont précis. Pour mieux situer la date du mémoire, on se rappellera que Pierre Boucher fut baptisê à Mortagne, Perche, France, le premier août $1622,{ }^{17}$ et que Madeleine Gaultier épousa Charles Petit de Livilliers à Montréal, le 29 août 1694.

A l'inventaire de René Gaultier, fait par le notaire Antoine Adhémar, à la demande de Marie Boucher, les $1^{\text {er }}$ et 2 août 1693, Louis est donné comme premier de ses huit enfants vivants, qui sont tous nommés par ordre de naissance. Rien de plus à son sujet. Il était encore au Canada.

Ajoutons une explication bien nécessaire sur le document suivant, que l'on trouve aux Archives Nationales de Paris, et qui concerne évidemment notre Louis:

Pièce No 3.

(Archives Nationales, Paris. - Colonies. - D2 C, vol. 222, fol. 813).

*Varennes de la Veranderie; enseigne, Canada, 1690; - confirmé, Canada, 16 mars 1691; - lieutenant réformé, Canada, 1692; - confirmé, Canada, 1er mars 1693 ; - mort, $1694 »$.

Cette date donnée pour la mort de Louis est évidemment une erreur et pose un problème, puisqu'on sait par d'autres documents, spécialement ceux que l'on verra ci-après, qu'il vivait en 1697, et qu'il mourut en 1706-1707.

16 Ce mémoire a été publié dans le BRH de 1926, pp. 398 à 404 . Il se trouve au Fonds Baby, à l'Université de Montréal.

17 On trouve l'acte de baptême de Pierre Boucher dans: R. P. Archange Godbout, O.F.M.: « Origine des familles canadiennes-françaises 》, p. 150. 
Il nous semble que l'auteur de la liste a tout simplement confondu deux Louis Gaultier de Varennes, et que la date de 1694 concerne l'ancien Louis Gaultier de Boumois, qui, après la perte du château et du titre de Boumois en 1671, aurait pris le titre de Louis Gaultier de Varennes. On sait, d'après le mémoire de Pierre Boucher cité plus haut, que Louis Gaultier de La Veranderie, sans doute à cause de la présence de son oncle Charles Gaultier de La Veranderie, avait remplacé son titre par celui de «de Varennes », et d'autres documents que nous verrons plus loin donnent aussi la preuve de ce changement. ${ }^{18}$

Louis Gaultier de Boumois devenu de Varennes était un cousin germain d'Adam-Pierre, et il se trouvait donc parent du 2 au 4 avec notre Louis Gaultier de La Veranderie devenu lui aussi de Varennes.

Louis Gaultier de Boumois et de Varennes nous est connu par plusieurs documents que nous avons mentionné ailleurs ${ }^{19}$ et dont nous verrons un des plus importants.

18 Louis Gaultier de la Veranderie porta ce titre depuis le 17 avril 1675 au moins, alors qu'il n'avait qu'un an et demi environ: "Donation par Jeanne Evrard (Esnard), veuve de Christophe Crevier de la Meslée, à Louis Gaultier de La Veranderie, d'une terre de dix par cent arpents, audessus de la ville des Trois-Rivières, sur le fleuve Saint-Laurent, venant d'une concession du 15 novembre 1658 ». derie ».

C'est la première mention que l'on a au Canada du titre de « La Veran-

Voir: Greffe de Sévérin Ameau, Cour Supérieure des Trois-Rivières.

- Cité dans: Inventaire des greffes des notaires, vol. XI, p. 102. bre-décembre 1956 .

Antonio Champagne, c.r.i.c.,

St-Boniface, Man.

(à suivre) 